LPT-ORSAY 01/07

NYU-TH 01/01/02

hep-th/0101234

\title{
The radion in brane cosmology
}

\author{
Pierre Binétruy*, Cédric Deffayet ${ }^{\dagger}$, David Langlois ${ }^{\dagger \dagger}$ \\ * LPT|, Université Paris-XI, Bâtiment 210, F-91405 Orsay Cedex, France; \\ $\dagger$ Department of Physics, New York University, \\ 4 Washington Place, New York, NY 10003, USA. \\ †† Institut d'Astrophysique de Paris, \\ Centre National de la Recherche Scientifique, \\ 98bis Boulevard Arago, 75014 Paris, France
}

\begin{abstract}
We consider the homogeneous cosmological radion, which we define as the interbrane distance in a two-brane and $Z_{2}$ symmetrical configuration. In a coordinate system where one of the brane is at rest, the junction conditions for the second (moving) brane give directly the (non-linear) equations of motion for the radion. We analyse the radion fluctuations and solve the non-linear dynamics in some simple cases of interest.
\end{abstract}

\footnotetext{
${ }^{1}$ Pierre.Binetruy@th.u-psud.fr, cjd2@physics.nyu.edu, langlois@iap.fr

${ }^{2}$ Unité mixte de recherche UMR ${ }^{o} 8627$.
} 


\section{Introduction}

Motivated by recent developments in string theory, the idea of confining matter in a three-brane embedded in a higher dimensional spacetime has stimulated lately a lot of activity in several fields of research. In cosmology, it has been shown that, quite generically, the matter confinement in a brane entails a non-standard evolution of the brane geometry [1]. In other words, the Friedmann equations governing the expansion of our 'apparent' universe are modified. This could lead to trouble because nucleosynthesis would give very different light element abundances with a modified scale factor evolution. Therefore, a strong constraint on brane models is the recovery of standard cosmological evolution at least since nucleosynthesis. A nice solution to this problem has been provided by applying the idea of the Randall-Sundrum model [2, 3] that a tension in the brane can be compensated by a negative cosmological constant in the bulk, as far as the internal brane expansion is concerned. Applied to cosmology, this idea leads to the recovery of the usual Friedmann law when the ordinary cosmological energy density is much less than the brane tension [4]. By contrast, at earlier times, when the energy density is higher than the tension one finds a non-standard evolution in the brane [5].

Another solution that has been suggested in the case of finite size extra-dimension (see e.g. [6] for the case of infinite extra-dimension) is to consider a stabilizing potential for the so-called radion [7, 8]. The precise definition of the radion is the source of some confusion in the literature. It is usually described as the metric component along the extra-dimension(s), but as emphasized in [9] it can be reinterpreted as the relative distance between the two branes. Stabilization of the radion can be explicitly realized [10] by adding some matter in the bulk, a scalar field for simplicity, so that the relative distance between the two branes will be stabilized. This mechanism has been applied [10, 11, 12, 13] to the two-brane model of Randall and Sundrum [2], in order to recover gravity consistent with the observations on the negative tension brane (without stabilization, one gets a Brans-Dicke gravity with a negative parameter [14]). In cosmology, this mechanism has been discussed in [15] and [16] but the claim that this would lead to the usual Friedmann equations has been challenged in 17]. Let

us mention that the radion has also been discussed in the context of holography [18. 
The purpose of the present work will not be to study the cosmological consequences of a stabilizing mechanism for the radion, task which will be left for future work, but more modestly to characterize the radion in cosmology (see also a brief discussion in [19]), to write down the equations of motion that govern its dynamics, which will be analysed in some simple examples.

Previous treatments of the radion in brane systems [9, 12, 20, 21] start from the beginning with a perturbative approach: the radion is thus hidden among the metric fluctuations, which have been the subject of active research lately in cosmology (see 222] and references therein). Our approach is quite different in the sense that the radion dynamics appears directly from the junction conditions. It also gives us the full non-linear dynamics of the homogeneous radion (i.e. ignoring the ordinary spatial dependence of the radion). We are thus able to show that the dynamics of the radion is more subtle than that of a scalar field coupled to the trace of the matter energy-momentum tensor.

Our plan is the following. We begin in section 2 by defining the coordinate system in which we describe the two-brane configuration. We then focus on the internal geometries inside the two branes in section 3. Section 4 is the central part of this paper, where the equations of motion of the radion are derived. Starting in section 5, we apply our general results to the case of an empty anti-de Sitter $\left(A d S_{5}\right)$ bulk spacetime. The last two sections are devoted to the analysis of the radion evolution in this geometry, first using a perturbative approach (section 6), then in the full non-linear case (section 7).

\section{The two-brane cosmological system}

The purpose of this section will be to present in a geometrical way the space-time configuration we wish to study. The spacetime will be supposed to be five-dimensional and to satisfy three-dimensional spatial homogeneity and isotropy in order to obtain (unperturbed) cosmological configurations. Two spatially homogeneous and isotropic three-branes live in this spacetime. The fifth dimension is periodic and we assume a mirror (orbifold) symmetry across each of the branes. The space between the two branes represents half of the periodic space along the fifth dimension, the other half being the mirror image with 
respect to any of the two branes.

In any coordinate system, even adapted to the three-dimensional homogeneity and isotropy, the two branes would be in general in motion along the fifth dimension. It turns out that it is possible, and convenient, to define the fifth dimensional coordinate $y$ as part of a Gaussian normal coordinate system with respect to one of the branes, chosen arbitrarily and called the reference brane. The reference brane is at rest in the fifth dimension, in $y=0$ say, and the spacetime metric in this coordinate system (which is not unique) is of the form

$$
d s^{2}=g_{A B} d x^{A} d x^{B}=-n(t, y)^{2} d t^{2}+a(t, y)^{2} \gamma_{i j} d x^{i} d x^{j}+d y^{2},
$$

where the $x^{i}$ are coordinates for the maximally symmetric threedimensional submanifolds, $t$ is a time coordinate. The metric components are necessarily of this form because of the symmetries, but their explicit dependence of $t$ and $y$ can be obtained only by solving the five-dimensional Einstein's equations (with the appropriate boundary conditions),

$$
G_{A B} \equiv R_{A B}-\frac{1}{2} R g_{A B}=\kappa^{2} T_{A B},
$$

where $R_{A B}$ is the Ricci tensor, $R$ its trace, and $T_{A B}$ the energymomentum tensor. The coupling of the matter to the geometry, $\kappa^{2}$, can be written as the inverse of the third power of the five dimensional reduced Planck mass.

If the reference brane is now at rest, the second brane will be in general moving in the same coordinate system. Its position at any time $t$ will be denoted

$$
y=\mathcal{R}(t)
$$

and we call this function $\mathcal{R}(t)$, which represents the relative distance between the two branes, the cosmological radion. Note that the rôle of the reference brane and of the second brane can of course be exchanged. Finally we wish to stress that here, unlike most of the literature, our radion is defined in a non-perturbative way. We will show later that our definition coincides, in the linearized approach, with the traditional definition of the radion.

\section{Induced geometries in the two branes}

The induced metric in the reference brane immediately follows from the spacetime metric (i]) and can be written in the usual Robertson- 
Walker form,

$$
d s_{\text {induced }}^{2}=-d t^{2}+a_{0}^{2} d \mathbf{x}^{2},
$$

where the time coordinate has been redefined such that $n(t, 0)=1$, and $a_{0}(t) \equiv a(t, y=0) . t$ is thus the proper time associated with the reference brane.

To get the induced geometry for the second brane, one must take into account its displacement in the fifth dimension, and one finds from (11) the induced line element

$$
d s_{\text {induced }}^{2}=-\left[n^{2}(t, \mathcal{R}(t))-\dot{\mathcal{R}}^{2}\right] d t^{2}+a^{2}(t, \mathcal{R}(t)) d \mathbf{x}^{2},
$$

where a dot will always stand for a (possibly partial) derivative with respect to $t$. This can be rewritten in the form

$$
d s_{\text {induced }}^{2}=-d \tau^{2}+a_{2}^{2}(\tau) d \mathbf{x}^{2},
$$

where $\tau$ is the second brane cosmic time (i.e. the proper time for a comoving observer) which is related to the reference brane cosmic time $t$ by

$$
d \tau=n(t, \mathcal{R}(t)) \sqrt{1-\frac{\dot{\mathcal{R}}^{2}}{n^{2}(t, \mathcal{R}(t))}} d t \equiv n_{2} \gamma^{-1} d t .
$$

And the scale factor in the second brane is simply

$$
a_{2}(t)=a(t, \mathcal{R}(t))
$$

or $a_{2}(\tau)$ if one expresses $t$ in terms of $\tau$.

It is also useful to introduce the expansion rate for the second brane, which can be defined as

$$
H_{2}(t) \equiv \frac{d a_{2} / d t}{a_{2}}=\left(\frac{\dot{a}}{a}+\frac{a^{\prime}}{a} \dot{\mathcal{R}}\right)_{2} .
$$

However, $\mathrm{H}_{2}$ does not coincide with the standard definition of the Hubble parameter for a cosmological observer in the second brane because it is defined with respect to the time $t$, which is the cosmic time in the reference brane but not in the second brane. The traditional Hubble parameter for the second brane corresponds to the definition

$$
\mathcal{H}_{2}(\tau) \equiv \frac{d a_{2} / d \tau}{a_{2}}=\frac{\gamma}{n_{2}} H_{2}
$$


Finally, one can introduce the unit velocity vector corresponding to the second brane, whose components read

$$
u^{A}=\left\{\frac{d t}{d \tau}, 0,0,0, \frac{d y}{d \tau}\right\}=n_{2}^{-1} \gamma\left\{1,0,0,0, \frac{d \mathcal{R}}{d t}\right\} .
$$

At this point, we can easily infer the cosmological evolution in the second brane from the knowledge of the cosmological evolution in the first brane and the position of the second brane with respect to the first. What will be studied next is the connection with the matter content of the second brane, which has been ignored up to this point.

\section{Junction conditions}

The matter content of the branes is directly related to the jump of the extrinsic curvature tensor across the brane. This relation has been established explicitly in the case of a brane at rest with respect to the coordinate system (1) in our previous works [1, 5]. We generalize our result to include the case of a brane moving with respect to the coordinate system.

We will now consider explicitly the second brane, but all the following expressions apply as well to the reference brane by taking $\mathcal{R}=0$. The extrinsic curvature tensor on the brane is defined by the expression

$$
K_{A B}=h_{A}^{C} \nabla_{C} n_{B}
$$

where $n^{A}$ is a unit vector field normal to the brane worlsheet and

$$
h_{A B}=g_{A B}-n_{A} n_{B}
$$

is the induced metric on the brane.

It is easy to compute the components of the unit normal vector from the components of the brane velocity (11), by using the fact that $n^{A}$ must satisfy the two following conditions:

$$
g_{A B} n^{A} n^{B}=1, \quad g_{A B} n^{A} u^{B}=0,
$$

and we get

$$
n^{A}=\left\{\frac{\dot{\mathcal{R}}}{n^{2} \sqrt{1-\frac{\dot{\mathcal{R}}^{2}}{n^{2}}}}, 0,0,0, \frac{1}{\sqrt{1-\frac{\dot{\mathcal{R}}^{2}}{n^{2}}}}\right\}
$$


Then, a straightforward calculation consisting in the substitution of the components (15) into the definition (12) yields the following (non zero) components for the extrinsic curvature tensor:

$$
\begin{aligned}
K_{0}^{0} & =\frac{\ddot{\mathcal{R}}+n n^{\prime}-2 \frac{n^{\prime}}{n} \dot{\mathcal{R}}^{2}-\frac{\dot{n}}{n} \dot{\mathcal{R}}}{n^{2}\left(1-\frac{\dot{\mathcal{R}}^{2}}{n^{2}}\right)^{5 / 2}} \\
K_{0}^{5} & =\dot{\mathcal{R}} K_{0}^{0}, \quad K_{5}^{0}=-K_{0}^{5} / n^{2}, \\
K_{j}^{i} & =\frac{1}{\sqrt{1-\frac{\dot{\mathcal{R}}^{2}}{n^{2}}}\left(\frac{a^{\prime}}{a}+\frac{\dot{a} \dot{\mathcal{R}}}{a n^{2}}\right) \delta_{j}^{i},} \\
K_{5}^{5} & =-\frac{\dot{\mathcal{R}}^{2}\left(\ddot{\mathcal{R}}+n n^{\prime}-2 \frac{n^{\prime}}{n} \dot{\mathcal{R}}^{2}-\frac{\dot{n}}{n} \dot{\mathcal{R}}\right)}{n^{4}\left(1-\frac{\dot{\mathcal{R}}^{2}}{n^{2}}\right)^{5 / 2}},
\end{aligned}
$$

where all coefficients take their value on the brane, i.e. at $t$ and $y=\mathcal{R}(t)$.

Let us now introduce the energy-momentum tensor on the second brane, which, because of the symmetries of the setup, is necessarily of the perfect fluid form

$$
S_{A B}^{(2)}=\left(\rho_{2}+P_{2}\right) u_{A} u_{B}+P_{2} h_{A B} .
$$

We also define $\hat{S}_{A B}$ as

$$
\hat{S}_{A B} \equiv S_{A B}-\frac{1}{3} S h_{A B}
$$

The junction conditions 23] for a hypersurface in a five-dimensional world read [1]

$$
\left[K_{\mu \nu}\right]=-\kappa^{2} \hat{S}_{\mu \nu},
$$

where the brackets stand for the jump across the brane, and $K_{\mu \nu}=$ $e_{\mu}^{A} e_{\nu}^{B} K_{A B}$ (and a similar expression for $\hat{S}_{\mu \nu}$ ) where the four vectors $e_{\mu}^{A}$ $(\mu=0,1,2,3)$ form a basis of the vector space tangent to the brane worldvolume. For the second brane, this gives explicitly

$$
\left[K_{\mu \nu}\right]_{2}=K_{\mu \nu}\left(t, \mathcal{R}(t)^{+}\right)-K_{\mu \nu}\left(t, \mathcal{R}(t)^{-}\right)=-2 K_{\mu \nu}(t, \mathcal{R}(t)),
$$

where the last equality follows from the identification of the point $y=\mathcal{R}(t)$ with the point $y=-\mathcal{R}(t)$ and from the $Z_{2}$ symmetry across the second brane. Note that, whereas all expressions (15-22) apply also 
to the reference brane (by taking $\mathcal{R}=0$ ), the last expression differs by a sign for the two branes: one would find $\left[K_{\mu \nu}\right]_{0}=2 K_{\mu \nu}(t, y=0)$ for the reference brane.

It is easy to compute the components of $\hat{S}_{A}^{B}$, using the velocity components (11) and one finds

$$
\begin{aligned}
\hat{S}_{0}^{0} & =-\frac{2 \rho_{2}+3 P_{2}}{3} \gamma^{2} \\
\hat{S}_{5}^{0} & =\frac{2 \rho_{2}+3 P_{2}}{3} \gamma^{2} \frac{\dot{\mathcal{R}}}{n^{2}}, \quad \hat{S}_{0}^{5}=-\frac{2 \rho_{2}+3 P_{2}}{3} \gamma^{2} \dot{\mathcal{R}} \\
\hat{S}_{j}^{i} & =\frac{1}{3} \rho_{2} \delta_{j}^{i} \\
\hat{S}_{5}^{5} & =\frac{2 \rho_{2}+3 P_{2}}{3} \gamma^{2} \frac{\dot{\mathcal{R}}^{2}}{n^{2}} .
\end{aligned}
$$

Equating this with the extrinsic curvature tensor, according to (22), all equations reduce to only two equations, which read

$$
\begin{aligned}
\frac{\ddot{\mathcal{R}}}{n^{2}}+\frac{n^{\prime}}{n}\left(1-2 \frac{\dot{\mathcal{R}}^{2}}{n^{2}}\right)-\frac{\dot{n}}{n} \frac{\dot{\mathcal{R}}}{n^{2}} & =-\frac{\kappa^{2}}{6}\left(2 \rho_{2}+3 P_{2}\right)\left(1-\frac{\dot{\mathcal{R}}^{2}}{n^{2}}\right)^{3 / 2} \\
\frac{a^{\prime}}{a}+\frac{\dot{a}}{a} \frac{\dot{\mathcal{R}}}{n^{2}} & =\frac{\kappa^{2}}{6} \rho_{2}\left(1-\frac{\dot{\mathcal{R}}^{2}}{n^{2}}\right)^{1 / 2}
\end{aligned}
$$

where, once more, all the metric coefficients take their value on the brane. The above system describes the full non-linear dynamics of the homogeneous cosmological radion. The non-linearity is manifest in the terms quadratic in $\dot{\mathcal{R}}$ but is also hidden in the fact that all the metric coefficients depend on $\mathcal{R}$.

It is interesting to note that the left hand side of equation (28) is simply the (covariant) acceleration in the fifth direction of a particle with the velocity (11). This can be understood by noticing that $u^{A} K_{A B} u^{B}=-n_{B} u^{C} \nabla_{C} u^{B}$, as a consequence of (12) and (14). $u^{A} K_{A B} u^{B}$, which is also $-K_{0}^{0}$, therefore vanishes for a geodesic motion. The right hand side of equation (28) will thus induce a deviation from geodesic motion for a "comoving" observer on the second brane (at fixed coordinate $x^{i}$ ).

The second order equation (28) can in fact be derived from the first order equation (29). Indeed, if one takes the time derivative of (29) and adds it to $\mathrm{H}_{2}$ times (29), one gets, after an overal multiplication 
by $\gamma^{-2}$, the expression

$$
\begin{aligned}
& H_{2}\left[\frac{\ddot{\mathcal{R}}}{n^{2}}+\frac{n^{\prime}}{n}\left(1-2 \frac{\dot{\mathcal{R}}^{2}}{n^{2}}\right)-\frac{\dot{n}}{n} \frac{\dot{\mathcal{R}}}{n^{2}}\right] \\
& -\frac{1}{3} G_{05}\left(1-\frac{\dot{\mathcal{R}}^{4}}{n^{4}}\right)-\frac{1}{3}\left(n^{-2} G_{00}+G_{55}\right)\left(1-\frac{\dot{\mathcal{R}}^{2}}{n^{2}}\right) \dot{\mathcal{R}} \\
= & -\frac{\kappa^{2}}{6} H_{2}\left(2 \rho_{2}+3 P_{2}\right)\left(1-\frac{\dot{\mathcal{R}}^{2}}{n^{2}}\right)^{3 / 2},
\end{aligned}
$$

where we have used

$$
\dot{\rho_{2}}=-3 H_{2}\left(\rho_{2}+P_{2}\right),
$$

which expresses the energy conservation on the second brane (assuming there is no inflow/outflow of energy from/to the bulk). A bulk energy-momentum tensor, satisfying the three-dimensional symmetries of homogeneity and isotropy, can be written in the form

$$
T_{A B}=\rho_{B} v_{A} v_{B}+P_{T}\left(h_{A B}^{(2)}+v_{A} v_{B}\right)+P_{B} h_{A B}^{(3)},
$$

where $v^{A}$ is a unit time-like vector (whose ordinary spatial components are zero) describing the fluid velocity, $P_{T}$ represents the transverse pressure (along the fifth dimension) and $P_{B}$ represents the pressure in the three ordinary dimensions; $h_{A B}^{(2)}$ is the projection tensor on the twodimensional $(t, y)$ subspace and $h_{A B}^{(3)}$ is the projection tensor on the three-dimensional ordinary space. Because of the $Z_{2}$ symmetry, the bulk fluid cannot go "across" the branes, and we must thus impose the further condition on the bulk fluid that the vector field $v^{A}$ coincides, on the branes, with the brane velocity $u^{A}$ ? . It is then not difficult to check explicitly that the second and third terms on the left hand side of (30), expressed in terms of $T_{A B}$ by use of Einstein's equations (2), just cancel. Equation (28) is thus derived, provided $H_{2}$ is not zero.

Equation (29) can be solved algebraically to express $\dot{\mathcal{R}}$ in terms of the other quantities. One finds

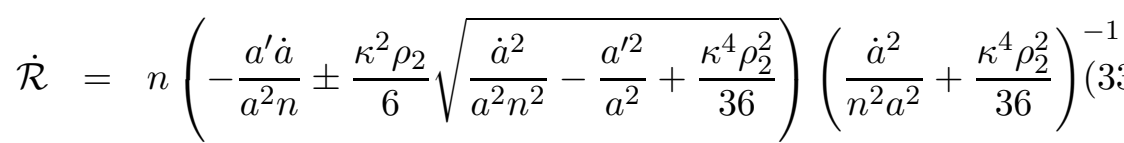

\footnotetext{
${ }^{3}$ Simultaneously $h_{A B}^{(2)}+v_{A} v_{B}=n_{A} n_{B}$.
} 
If $a^{\prime} / a$ and $\rho_{2}$ are of the opposite sign, then (29) has one solution if $|\dot{a} /(a n)|>\left|a^{\prime} / a\right|$, no solution otherwise. If $a^{\prime} / a$ and $\rho_{2}$ are of the same sign, then (29) has two solutions when $\mid \dot{a} /($ an $)|<| a^{\prime} / a \mid$, and only one solution otherwise. In both cases, when there is a unique solution, it corresponds to the root in (33) with the sign being the same as that of $\dot{a} / a$.

To conclude this section, let us mention that the equations of motion for the radion can be rewritten in terms of the cosmic time on the second brane, $\tau$, instead of the cosmic time on the reference brane $t$ :

$$
\begin{aligned}
& \mathcal{R}_{, \tau \tau}+\frac{n^{\prime}}{n}\left(1+\mathcal{R}_{, \tau}^{2}\right)=-\frac{\kappa^{2}}{6}\left(2 \rho_{2}+3 P_{2}\right) \sqrt{1+\mathcal{R}_{, \tau}^{2}}, \\
& \frac{a^{\prime}}{a}+\frac{\dot{a}}{a n} \frac{\mathcal{R}_{, \tau}}{\sqrt{1+\mathcal{R}_{, \tau}^{2}}}=\frac{\kappa^{2}}{6} \rho_{2}\left(1+\mathcal{R}_{, \tau}^{2}\right)^{-1 / 2} .
\end{aligned}
$$

The second equation has been obtained by [24] in the particular case where the reference brane is Minkowski (hence $\dot{a} / a=0$ and $\rho_{2}$ is a constant.

\section{Radion dynamics in an $A d S_{5}$ bulk spacetime}

In order to explore the dynamical evolution of the radion, we now need to be more specific and to resort to explicit expressions for the spacetime metric (11). We will thus choose to work with an $A d S_{5}$ bulk spacetime, for which it is possible to recover (approximately) [5], at late times, standard cosmology, and which corresponds to the cosmological, i.e. non static, generalization of the Randall-Sundrum model [3].

Keeping aside the two branes, we assume that the bulk spacetime is empty, except for a negative cosmological constant $\Lambda$. It is useful to define a mass scale $\mu$ associated with $\Lambda$, according to the expression

$$
\mu=\sqrt{-\Lambda / 6}
$$

( $\mu^{-1}$ is the $A d S_{5}$ curvature radius) and an energy density scale $\sigma$, defined by

$$
\sigma=6 \frac{\mu}{\kappa^{2}}
$$


Note that, in the Randall-Sundrum model [3], the energy density in the brane is supposed to be exactly this $\sigma$. In the present work, we consider any value for the energy densities in the two branes.

We will also assume that the five-dimensional Weyl tensor is zero. Our global spacetime is therefore made of two pieces of $A d S_{5}$ separated by the two branes. Assuming the usual $Z_{2}$ symmetry with respect to each brane, the two $A d S_{5}$ pieces are then simply mirror symmetric.

In [5], we have solved explicitly the five-dimensional Einstein's equations

$$
G_{A B}-\Lambda g_{A B}=\kappa^{2} T_{A B},
$$

in the case where the only matter in spacetime is the one confined in a brane. We obtained the following form for the component of (11):

$$
\begin{aligned}
& a(t, y)=a_{0}(t)\left(\cosh \mu y-\eta_{0} \sinh \mu|y|\right) \\
& n(t, y)=\cosh \mu y-\tilde{\eta}_{0} \sinh \mu|y|,
\end{aligned}
$$

where $\eta_{0}$ and $\tilde{\eta}_{0}$ are two functions of time, related to the energy density in the reference brane by

$$
\eta_{0}(t)=\frac{\rho_{0}(t)}{\sigma}, \quad \tilde{\eta}_{0}(t)=\eta_{0}+\frac{\dot{\eta}_{0}}{H_{0}}
$$

where $H_{0} \equiv \dot{a}_{0} / a_{0}$ is the Hubble parameter. For future use we also define $\eta_{2}$ as

$$
\eta_{2}(t)=\frac{\rho_{2}(t)}{\sigma}
$$

where $\rho_{2}(t)$ is the energy density of the second brane. It must be stressed that, whereas the functional form of the metric components (39,40) is obtained by solving the Einstein equations in the bulk, the relationship between the coefficients and the reference brane matter given in (41) comes from the junction conditions [1] across the reference brane.

Finally, the evolution of the scale factor $a_{0}(t)$ and of the energy density $\rho_{0}(t)$ is determined by solving the coupled system consisting of the generalized Friedmann equation

$$
H_{0}^{2}=\frac{\Lambda}{6}+\frac{\kappa^{4}}{36} \rho_{0}^{2}-\frac{k}{a_{0}^{2}}=\mu^{2}\left(\eta_{0}^{2}-1\right)-\frac{k}{a_{0}^{2}}
$$

where $k=-1,0,1$ according to the three-dimensional spatial curvature (the term $\mathcal{C} / a_{0}^{4}$ does not appear because we have assumed a 
vanishing Weyl tensor) and the usual energy conservation law

$$
\dot{\rho}_{0}=-3 H_{0}\left(\rho_{0}+P_{0}\right) \text {. }
$$

A useful identity is an integral of Einstein's equations

$$
\left(\frac{\dot{a}}{n a}\right)^{2}=\frac{1}{6} \kappa^{2} \rho_{B}+\left(\frac{a^{\prime}}{a}\right)^{2}-\frac{k}{a^{2}}
$$

which was obtained in [5]. The generalized Friedmann equation (43) on the reference brane is simply the application of this identity (with the junction conditions) in $y=0$.

The fully non-linear evolution of the radion, coupled to the evolution of the matter on the two branes, is thus given by the first-order

system of differential equations consisting of (43), (44), (31) and (33), which can be rewritten in the simpler form

$$
\dot{\mathcal{R}}=n\left(-\frac{a^{\prime} \dot{a}}{a^{2} n} \pm \frac{\kappa^{2} \rho_{2}}{6}\left|\mathcal{H}_{2}\right|\right)\left(\frac{\dot{a}^{2}}{n^{2} a^{2}}+\frac{\kappa^{4} \rho_{2}^{2}}{36}\right)^{-1},
$$

where $\mathcal{H}_{2}$ is the Hubble parameter of the second brane defined in (10).

One can also check, starting from the expression (9) in terms of the metric component (39) and using (29) and (43), all terms in $\dot{\mathcal{R}}$ cancel and one recovers the generalized Friedmann equation on the second brane, that is

$$
\mathcal{H}_{2}^{2}=\frac{\Lambda}{6}+\frac{\kappa^{4}}{36} \rho_{2}^{2}-\frac{k}{a_{2}^{2}}=\mu^{2}\left(\eta_{2}^{2}-1\right)-\frac{k}{a_{2}^{2}} .
$$

It has to be so because the generalized Friedmann equations comes from a purely local analysis, and the rôle of the two branes can be exchanged.

\section{Radion fluctuations}

In this section, we study perturbatively the dynamics of the radion fluctuations. We first define the background solution as the case where the radion is frozen, i.e. does not depend on time. This means that the second brane is at rest with respect to the first brane, i.e.

$$
y_{2}(t)=\overline{\mathcal{R}},
$$


where $\overline{\mathcal{R}}$ is a constant. One can then find explicit solutions of the Einstein's equations for the whole two-brane system. However, to ensure the continuity of the metric along the compact fifth dimension, one finds that the matter content in the second brane is necessarily related to the matter content of the first brane. This result was already noticed for a vanishing bulk cosmological constant [1]. We generalize it here to the case of a non-zero bulk cosmological constant.

If $\mathcal{R}$ is constant, then the system (28,29) simplifies considerably to yield

$$
\left(\frac{n^{\prime}}{n}\right)_{2}=-\frac{\kappa^{2}}{6}\left(2 \overline{\rho_{2}}+3 \overline{P_{2}}\right)
$$

and

$$
\left(\frac{a^{\prime}}{a}\right)_{2}=\frac{\kappa^{2}}{6} \overline{\rho_{2}}
$$

Substituting the explicit expressions (39) and (40) for $a$ and $n$, which depend on the energy content on the first brane, one can express the energy density and pressure of the second brane as functions of the energy density and pressure on the first brane and of the position $\mathcal{R}$. One finds

$$
\kappa^{2} \bar{\rho}_{2}(t)=6 \mu \frac{\sinh \mu \overline{\mathcal{R}}-\eta_{0} \cosh \mu \overline{\mathcal{R}}}{\cosh \mu \overline{\mathcal{R}}-\eta_{0} \sinh \mu \overline{\mathcal{R}}}
$$

and

$$
\kappa^{2} \bar{P}_{2}(t)=-2 \mu \frac{\sinh \mu \overline{\mathcal{R}}-\tilde{\eta}_{0} \cosh \mu \overline{\mathcal{R}}}{\cosh \mu \overline{\mathcal{R}}-\tilde{\eta}_{0} \sinh \mu \overline{\mathcal{R}}}-\frac{2}{3} \kappa^{2} \overline{\rho_{2}}
$$

Note that if one considers an equation of state of the form $P_{0}=$ $w_{0} \rho_{0}$ with $w_{0}$ constant for the matter on the reference brane (which includes the standard cases of radiation, non-relativistic matter and a cosmological constant), the equation of state of the matter on the second brane, which is completely determined here as a function of the relative distance between the two branes, will correspond to an equation of state $\bar{P}_{2}=w_{2} \bar{\rho}_{2}$ where, in general, $w_{2}$ is time dependent. Indeed $\eta_{0}$ and

$$
\tilde{\eta}_{0}=-\left(2+3 w_{0}\right) \eta_{0}
$$

are time dependent in general.

One exception is when the reference brane contains only a cosmological constant, i.e. $w_{0}=-1$, then one also gets $w_{2}=-1$ on the second brane. There is in this case an interesting correspondence between the cosmological constants on the two branes. Introducing the 
effective cosmological constant from the brane point of view as

$$
\lambda=\frac{\Lambda}{6}+\frac{\kappa^{4}}{36} \rho=\mu^{2}\left(\eta^{2}-1\right),
$$

we have

$$
\lambda_{2}=\frac{\lambda_{0}}{\left(\cosh \mu \overline{\mathcal{R}}-\eta_{0} \sinh \mu \overline{\mathcal{R}}\right)^{2}} .
$$

Thus, the two branes are simultaneously $d S_{4}(\lambda>0,|\eta|>1), M_{4}$ $(\lambda=0,|\eta|=1)$ or $A d S_{4}(\lambda<0,|\eta|<1)$ [25].

Another interesting case is when the reference brane undergoes ordinary cosmology, which occurs when $\eta_{0}$ is very small. One can then neglect $\eta_{0}$ as well as $\tilde{\eta}_{0}$ in the expressions (51-52) and the matter on the second brane reduces effectively to a cosmological constant.

In order to obtain the equation of motion for the radion fluctuations, we linearize the exact equations of motion (28-29) about the equilibrium configuration we have just defined. The linearized system reads

$$
\begin{aligned}
\frac{\delta \ddot{\mathcal{R}}}{n^{2}}+\delta\left(\frac{n^{\prime}}{n}\right)-\frac{\dot{n}}{n} \frac{\delta \mathcal{\mathcal { R }}}{n^{2}} & =-\frac{\kappa^{2}}{6}\left(2 \delta \rho_{2}+3 \delta P_{2}\right) \\
\delta\left(\frac{a^{\prime}}{a}\right)+\frac{\dot{a}}{a} \frac{\delta \dot{\mathcal{R}}}{n^{2}} & =\frac{\kappa^{2}}{6} \delta \rho_{2},
\end{aligned}
$$

where we recall that all metric coefficients take their value on the second brane. Furthermore, one can use the explicit expressions (3940) to find

$$
\begin{aligned}
& \delta\left(\frac{n^{\prime}}{n}\right)=m_{n}^{2} \delta \mathcal{R} \equiv\left[\mu^{2}-\frac{\kappa^{4}}{36}\left(2 \overline{\rho_{2}}+3 \overline{P_{2}}\right)^{2}\right] \delta \mathcal{R} \\
& \delta\left(\frac{a^{\prime}}{a}\right)=m_{a}^{2} \delta \mathcal{R} \equiv\left[\mu^{2}-\frac{\kappa^{4}}{36}{\overline{\rho_{2}}}^{2}\right] \delta \mathcal{R},
\end{aligned}
$$

where we have introduced two effective squared masses $m_{n}^{2}$ and $m_{a}^{2}$. Note that both $m_{n}$ and $m_{a}$ vanish in the particular case where the Randall-Sundrum condition between the tension and the bulk cosmological constant is satisfied on the second brane.

If one adds (56) with three times (57), one obtains the equation of motion

$$
\frac{\ddot{\delta \mathcal{R}}}{n^{2}}+\left(3 \frac{\dot{a}}{a}-\frac{\dot{n}}{n}\right) \frac{\delta \dot{\mathcal{R}}}{n^{2}}+\left(m_{n}^{2}+3 m_{a}^{2}\right) \delta \mathcal{R}=-\frac{\kappa^{2}}{6} \delta T_{2}
$$


where $\delta T_{2} \equiv-\delta \rho_{2}+3 \delta P_{2}$ is the linear perturbation of the trace of the matter energy-momentum tensor. One thus recognizes the equation of motion for a scalar field coupled to the (perturbed) trace of the matter energy-momentum tensor. The friction coefficient is the usual one (rewriting in terms of $\tau$ would give the natural $3 \mathcal{H}_{2}$ friction coefficient, as can be seen when extracting this equation directly from (34), (35)), but the mass term is an effective mass that depends both on the bulk cosmological constant and on the background matter content of the second brane.

This effective mass is time-dependent except in the case where the second brane matter has for equation of state $P_{2}=-\rho_{2}$. The two squared masses are then identical and equal to

$$
m_{a}^{2}=m_{n}^{2}=-\lambda_{2}
$$

where $\lambda_{2}$ is the effective cosmological constant from the brane point of view defined in (54). One thus recovers exactly previous results [9, 20, 21]. An immediate consequence of this is that when the first brane is $d S_{4}$, the small perturbations around the equilibrium position of the second brane (then also $d S_{4}$ ) are unstable, whereas when the first brane is $A d S_{4}$ (the second brane is then also $A d S_{4}$ ) they are stable. We will see in section 7 that this perturbative analysis matches with the exact non linear evolution.

The evolution of the radion fluctuations is entangled with the matter fluctuations. It is however possible when the matter fluctuations are of the adiabatic type, i.e.

$$
\delta P_{2}=c_{s 2}^{2} \delta \rho_{2}
$$

to derive an equation for only the radion fluctuations by considering the appropriate linear combination of (56) and (57),

$$
\frac{\check{\delta}}{n^{2}}+\left[\left(2+3 c_{s 2}^{2}\right) \frac{\dot{a}}{a n^{2}}-\frac{\dot{n}}{n^{3}}\right] \delta \dot{\mathcal{R}}+\left[m_{n}^{2}+\left(2+3 c_{s 2}^{2}\right) m_{a}^{2}\right] \delta \mathcal{R}=0 .
$$

The evolution of the matter fluctuations is then related to the radion fluctuation by (57), i.e.

$$
\frac{\kappa^{2}}{6} \delta \rho_{2}=\frac{\dot{a}}{a} \frac{\delta \mathcal{R}}{n^{2}}+m_{a}^{2} \delta \mathcal{R} .
$$


Assuming an equation of state $\overline{P_{2}}=w_{2} \overline{\rho_{2}}$ (and adiabatic fluctuations, i.e. $c_{s 2}^{2}=w_{2}$ ), the equation of motion (63) takes the form, after substitution of $m_{a}^{2}$ and $m_{n}^{2}$ by their explicit expressions,

$\delta \mathcal{R}_{, \tau \tau}+\left(2+3 w_{2}\right) \mathcal{H}_{2} \delta \mathcal{R}, \tau+3\left(1+w_{2}\right)\left(\mu^{2}-\frac{\kappa^{4}}{36}{\overline{\rho_{2}}}^{2}\left(2+3 w_{2}\right)\right) \delta \mathcal{R}=0$

where we have used here the second brane cosmic time $\tau$. One can solve this equation for de Sitter $\left(w_{2}=-1\right)$ on the two branes and one gets

$$
\delta \mathcal{R}=\delta \mathcal{R}_{i} e^{\mathcal{H}_{2}\left(\tau-\tau_{i}\right)}-\frac{\kappa^{2}}{6 \mathcal{H}_{2}^{2}} \delta \rho_{2 i}\left(1-e^{\mathcal{H}_{2}\left(\tau-\tau_{i}\right)}\right),
$$

where $\delta \mathcal{R}_{i}$ is the initial radion fluctuation and $\delta \rho_{2 i}$ is the initial density fluctuation (at time $\tau_{i}$ ). For de Sitter brane cosmology, we recover the instability mentioned above.

Note that in the case of the radion fluctuations in the strict RandallSundrum model, studied in [9], the friction coefficient in the above equation vanishes because the background is static, and both the effective squared masses $m_{a}^{2}$ and $m_{n}^{2}$ also vanish because of the RS condition relating the bulk cosmological constant and the brane tensions. One thus ends up with the equation of motion for a free scalar field.

\section{Non linear evolution of the radion}

In this section, we explore the non-perturbative aspects of the radion evolution in some simple cases, leaving a more systematic analysis for future work. We are going to consider the cases where the matter on the reference brane behaves like a cosmological constant. In these simple cases, the reference brane matter does not evolve, i.e. $\dot{\eta}_{0}=0$, and the metric components are separable and can be written in the form

$$
n(t, y)=n(y), \quad a(t, y)=a_{0}(t) n(y) .
$$

The explicit form of $n(y)$ depends on the sign of $\eta_{0}-1$. If $\eta_{0}>1$, the brane is $d S_{4}$; if $\eta_{0}<1$, the brane is $A d S_{4}$; and the case $\eta_{0}=1$ corresponds to the Randall-Sundrum model [3]. We will then further specialize to the cases where the brane matter of the second also behaves as a cosmological constant, implying $\dot{\eta}_{2}=0$. 


\section{$7.1 d S_{4}$ brane}

For a $d S_{4}$ reference brane, the dependence of the metric components on the fifth coordinate is simply (see e.g. [26])

$$
n(y)=\sqrt{\eta_{0}^{2}-1} \sinh \mu\left(y_{\mathrm{h}}-|y|\right),
$$

where

$$
y_{\mathrm{h}}=\frac{1}{\mu} \arg \operatorname{coth} \eta_{0}
$$

Here we suppose that the second brane is always located at $\mathcal{R}<y_{\mathrm{h}}$. Otherwise, there would be a horizon at $\mathcal{R}=y_{\mathrm{h}}$.

Equation (33) can then be rewritten in terms of dimensionless quantities in the form

$x_{, s}=-\mathcal{K}_{0}^{2} \sinh x \frac{h_{0} \cosh x \pm \eta_{2} \sinh x \sqrt{\eta_{2}^{2} \mathcal{K}_{0}^{2} \sinh ^{2} x-\mathcal{K}_{0}^{2} \cosh ^{2} x+h_{0}^{2}}}{h_{0}^{2}+\eta_{2}^{2} \mathcal{K}_{0}^{2} \sinh ^{2} x}$

where we have introduced the dimensionless distance of the second brane to the horizon $x$ and the dimensionless time $s$

$$
x \equiv \mu\left(y_{\mathrm{h}}-\mathcal{R}\right), \quad s=\mu t .
$$

The dimensionless Hubble parameter $h_{0}$ is defined by

$$
h_{0} \equiv \frac{1}{a_{0}} \frac{d a_{0}}{d s}=\frac{H_{0}}{\mu},
$$

and $\mathcal{K}_{0}$ is defined by

$$
\mathcal{K}_{0}=\sqrt{\left|\eta_{0}^{2}-1\right|}
$$

In general equation (70) is non separable since both $\eta_{2}$ and $h_{0}$ are function of time. It gets however simplified when one assumes that the energy momentum tensor of the second brane is that of a cosmological constant (implying $\dot{\eta}_{2}=0$ ), and if one assumes moreover that $k=0$ (in order to account for a slicing of $d S_{4}$ by flat 3 -spaces) so that $h_{0}=\mathcal{K}_{0}^{2}$ (as can be seen from (430). In this case equation (70) reads

$$
x_{, s}=-\sqrt{\eta_{0}^{2}-1} \sinh x \frac{\cosh x \pm \eta_{2} \sqrt{\eta_{2}^{2}-1} \sinh ^{2} x}{1+\eta_{2}^{2} \sinh ^{2} x},
$$


it becomes separable and can be solved analytically. Since the matter energy densities are frozen on both branes, the evolution of the radion is fully embodied in this unique first order equation. The evolution of the radion is thus completely determined by the knowledge of the initial position of the second brane, which we call $\mathcal{R}_{i}$. If the initial position $\mathcal{R}_{i}$ is located at the equilibrium position

$$
\bar{x}=\mu\left(y_{\mathrm{h}}-\overline{\mathcal{R}}\right)=-\arg \operatorname{coth} \eta_{2},
$$

then the brane does not move. However, if the brane is initially away from the equilibrium position, it moves. According to the perturbative analysis, a brane slightly off the equilibrium position moves further away since we found that the radion is unstable.

Here, we can determine the non-linear evolution. We have solved numerically the evolution of the radion in the cases where the brane is initially on the left $\left(\mathcal{R}_{i}<\overline{\mathcal{R}}\right)$ and on the right $\left(\mathcal{R}_{i}>\overline{\mathcal{R}}\right)$ of the equilibrium position. We observe the expected unstable behaviour. In the first case, illustrated in Fig. 1, the brane moves towards the reference brane until it collides with it (numerically it goes across it). In the second case, illustrated in Fig. 2, the brane moves further away but its motion freezes asymptotically at the horizon, as seen in the reference brane proper time. However, as can be seen from (7) and (74), this takes only a finite amount of the second brane proper time, as is typical for the crossing of a (Rindler or black hole) horizon.

One may obtain a simple analytical expression which illustrates this behaviour in the case of a empty second brane : $\eta_{2}=0$. The solution is

$$
x=\arg \tanh \left[\tanh x_{i} \exp \left(-\sqrt{\eta_{0}^{2}-1}\left(s-s_{i}\right)\right)\right],
$$

where $x_{i}$ is the initial position at time $s_{i}$. This is also the solution of (74) for non zero $\eta_{2}$ when the second brane approaches the horizon (i.e. for $x$ small).

\section{$7.2 \quad A d S_{4}$ brane}

For an $A d S_{4}$ reference brane one finds for the metric component

$$
n(y)=\sqrt{1-\eta_{0}^{2}} \cosh \mu\left(|y|-y_{c}\right)
$$




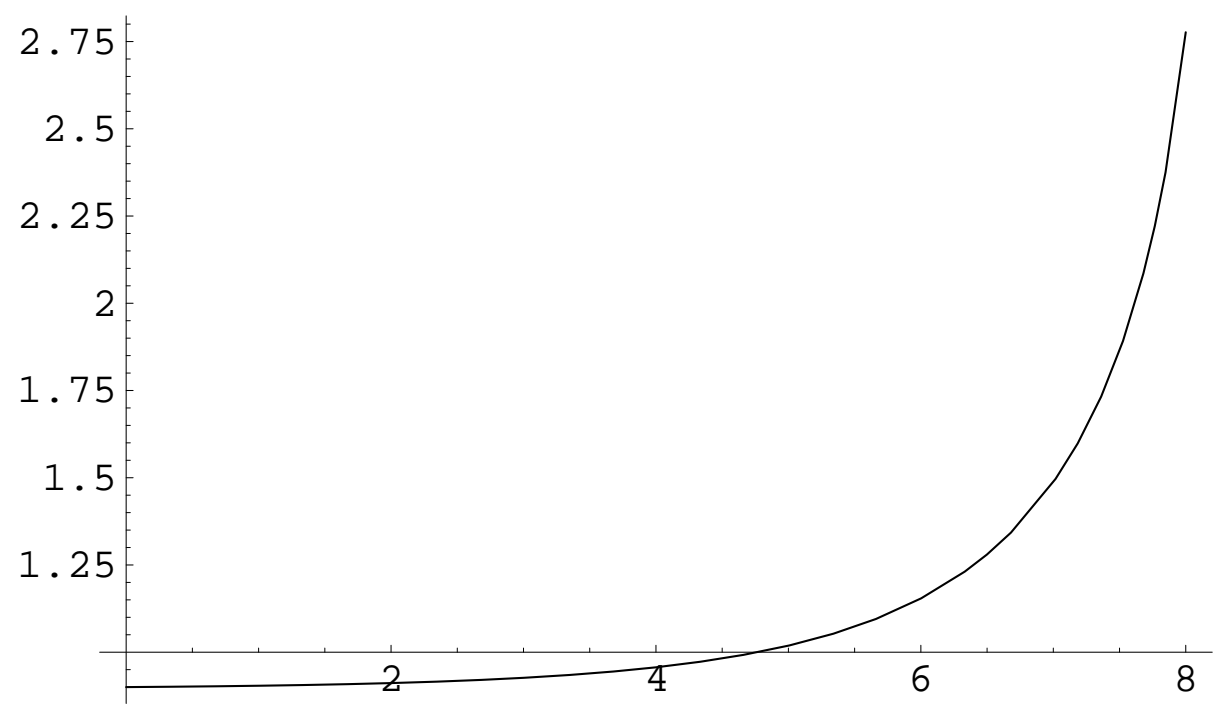

Figure 1: Evolution with time of the distance $x$ from the second brane to the "horizon" in the case of $d S_{4}$ for an initial position $\mathcal{R}_{i}<\overline{\mathcal{R}}$.

where

$$
y_{c}=\frac{1}{\mu} \arg \tanh \eta_{0} .
$$

In this case the warp factor $n$ has a minimum in $y=y_{c}$ enabling to have a configuration with two static branes of positive tensions 25] (see also [21, 27]). In any case, equation (33) reads

$x_{, s}=\mathcal{K}_{0}^{2} \cosh x \frac{-h_{0} \sinh x \pm \eta_{2} \cosh x \sqrt{\eta_{2}^{2} \mathcal{K}_{0}^{2} \cosh ^{2} x-\mathcal{K}_{0}^{2} \sinh ^{2} x+h_{0}^{2}}}{h_{0}^{2}+\eta_{2}^{2} \mathcal{K}_{0}^{2} \cosh ^{2} x}$,

where $x$ is defined by

$$
x \equiv \mu\left(\mathcal{R}-y_{c}\right),
$$

and $s$ and $\mathcal{K}_{0}$ are defined respectively as in (71) and (73). The scale factor is given straighforwardly by solving (43); one finds $a_{0}=$ $\sin \left(\mathcal{K}_{0} s\right) / \mu \mathcal{K}_{0}$, so that in this FLRW parametrization of $A d S_{4}$ there are coordinate singularities in $\mathcal{K}_{0} s=0$ and $\mathcal{K}_{0} s=\pi$. The dimensionless Hubble parameter is then $h_{0}=\mathcal{K}_{0} \operatorname{cotan}\left(\mathcal{K}_{0} s\right)$. The equation of motion (79) remains non separable even in the simple case where 


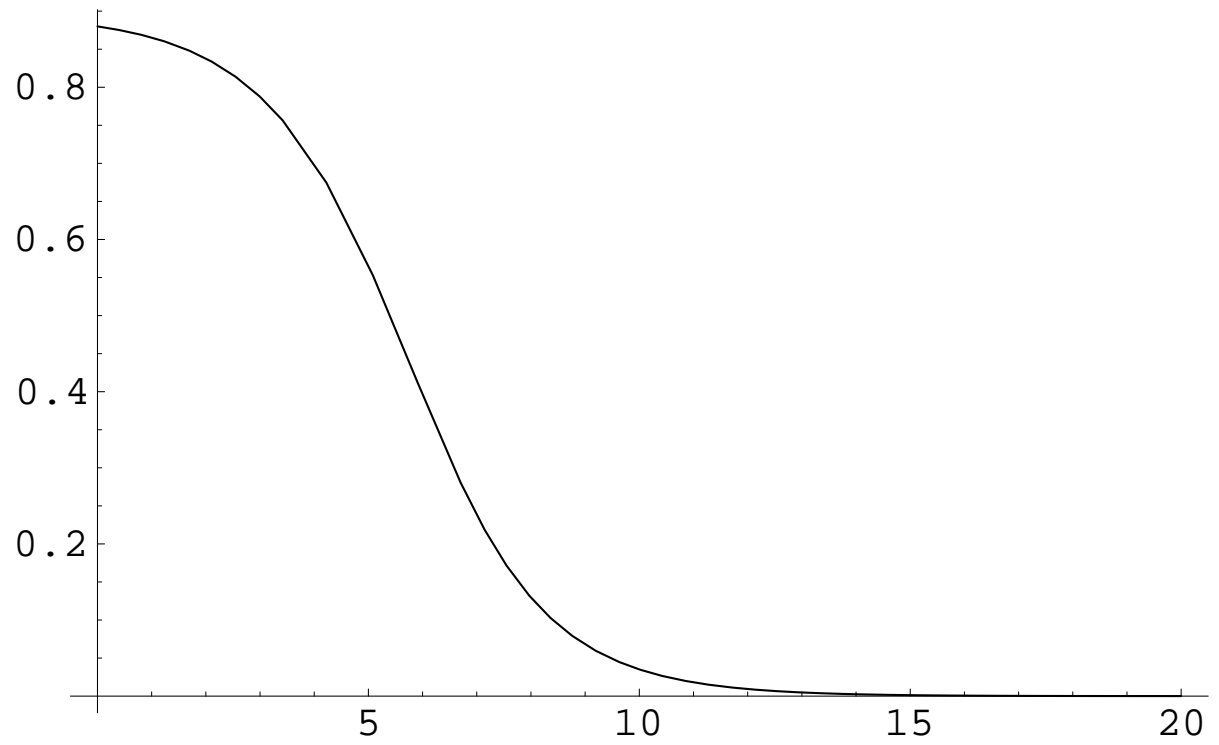

Figure 2: Evolution with time of the distance $x$ from the second brane to the "horizon" in the case of $d S_{4}$ for an initial position $\mathcal{R}_{i}>\overline{\mathcal{R}}$. 
$\dot{\eta_{2}}=0$, with $\eta_{2} \neq 0$. We have solved numerically for the evolution of the radion in the cases where the brane (with $\eta_{2}$ being constant and positive) is initially (at time $s_{i}$ such that $\mathcal{K}_{0} s_{i}<\pi / 2$ ) on the left $\left(x_{i}<\bar{x}\right)$ and on the right $\left(x_{i}>\bar{x}\right)$ of the equilibrium position $\bar{x}$ defined by

$$
\bar{x}=\mu\left(\overline{\mathcal{R}}-y_{c}\right)=\arg \tanh \eta_{2} .
$$

For small enough $s_{i}$, the only acceptable solution is given by taking the + sign in front of the square root in equation (79), as can be seen using the arguments given at the end of section 4 .

In both cases $\left(x_{i}<\bar{x}\right.$ and $\left.x_{i}>\bar{x}\right)$, illustrated in figures 3 and 4 , the brane first moves back toward its equilibrium position, and then reaches a point (at time $s_{c}$ close to $\pi / 2 \mathcal{K}_{0}$ ) where the square root equation (79) vanishes. The solution obtained from (79) with the + sign in front of the square root must then be matched (after time $s_{c}$ ) to a solution of (79) with a - sign is taken in front of the square root (the two solutions have the same tangent in $s_{c}$ ). Figures 3 and 19 are obtained with this matching.

Equation (79) becomes separable, and can be easily integrated, in the limiting case where the second brane is empty i.e. $\eta_{2}=0$. In this case, one finds:

$$
x=\arg \tanh \left[\tanh x_{i} \frac{\cos \left(\mathcal{K}_{0} s\right)}{\cos \left(\mathcal{K}_{0} s_{i}\right)}\right] .
$$

\section{3 $\quad M_{4}$ brane}

When the reference brane is Minkowskian ( $\eta_{0}$ being 1 , and $a=n=$ $\exp (-\mu|y|))$, equation (33) is given by

$$
x_{, s}= \pm \frac{e^{-x}}{\eta_{2}} \sqrt{\eta_{2}^{2}-1}
$$

where $x$ is given here by

$$
x \equiv \mu \mathcal{R}
$$

and $s$ is defined as above. The second brane equilibrium condition is simply given by the Randall-Sundrum [2, 3] matching condition expressed here as $\eta_{2}=-1$. For this particular $\eta_{2}$, one sees easily

\footnotetext{
${ }^{4}$ which is also the determinant of the quadratic equation obtained from equation (29)
} 


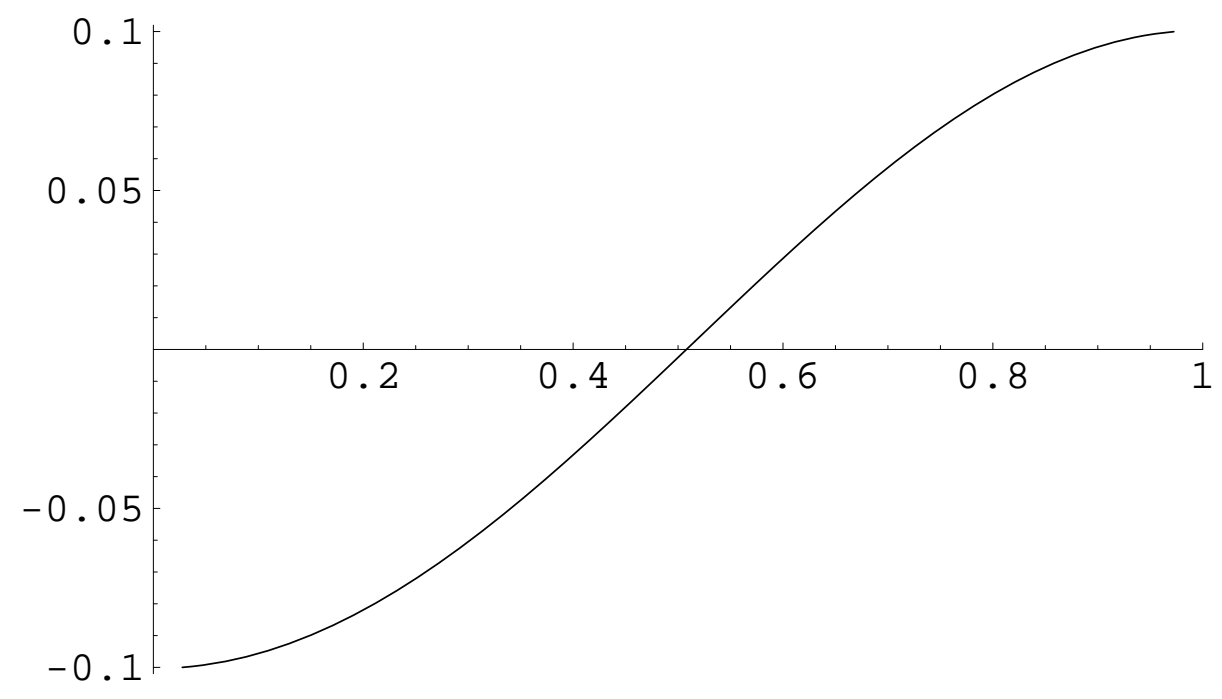

Figure 3: Evolution of the distance $x-\bar{x}$ between the brane position and the equilibrium position as a function of $\mathcal{K}_{0} s / \pi$, in the case of $A d S_{4}$ for an initial position $x_{i}<\bar{x}$. 


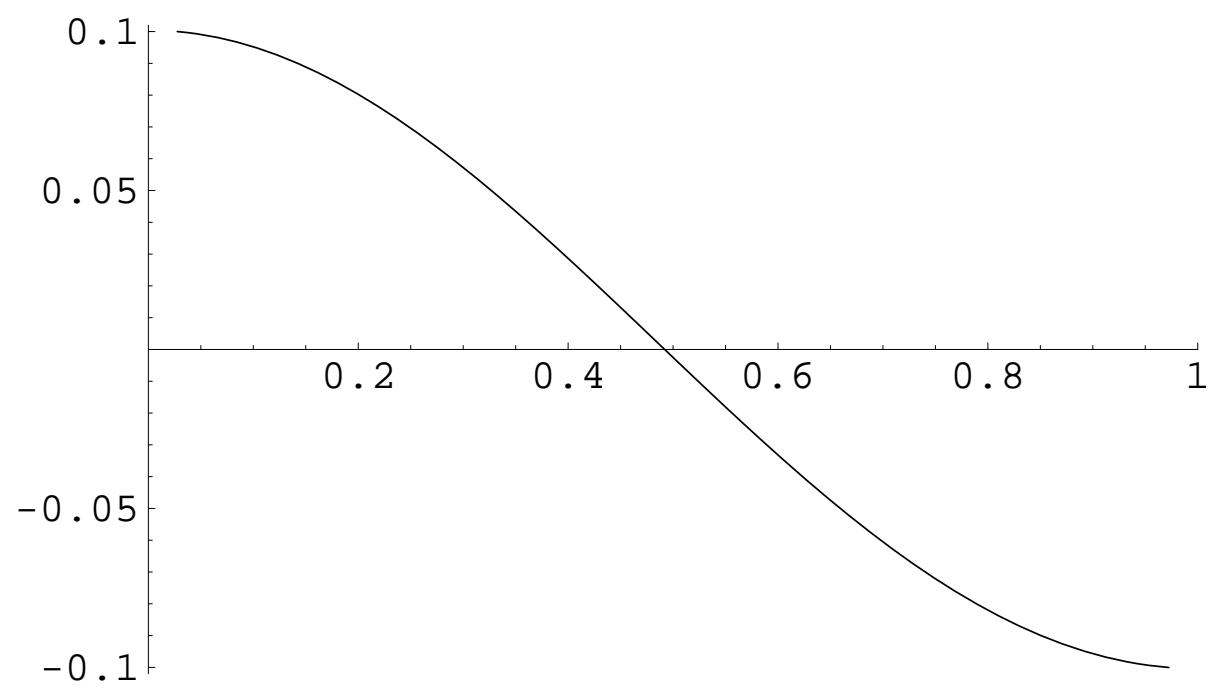

Figure 4: Evolution of the distance $x-\bar{x}$ between the brane position and the equilibrium position as a function of $\mathcal{K}_{0} s / \pi$, in the case of $A d S_{4}$ for an initial position $x_{i}>\bar{x}$. 
from equation (83) that the second brane must be at rest with respect to the first one, but that the equilibrium position $\bar{x}$ can be chosen arbitrarily. However a small departure from the equilibrium condition $\eta_{2}=-1$ results in either a colliding of the two branes or a runaway behaviour.

\section{Acknowledgments}

We thank Arthur Lue, who helped us to understand some subtleties associated with the numerical integration of equation (79). The work of C.D. is sponsored in part by NSF Award PHY 9803174, and by David and Lucile Packard Foundation Fellowship 99-1462.

\section{References}

[1] P.Binétruy, C.Deffayet, D.Langlois, Nucl. Phys. B 565, 269 (2000) hep-th/9905012.

[2] L. Randall and R. Sundrum, Phys. Rev. Lett. 83 (1999) 3370 hep-ph/9905221.

[3] L.Randall, R.Sundrum, Phys. Rev. Lett. 83, 4690 (1999) hepth/9906064.

[4] C. Csáki, M. Graesser, C. Kolda, J. Terning, Phys. Lett. B462, 34 (1999); J.M. Cline, C.Grojean, G.Servant, Phys. Rev. Lett. 83, 4245 (1999).

[5] P. Binétruy, C. Deffayet, U. Ellwanger, D. Langlois, Phys.Lett.B 477, 285 (2000) hep-th/9910219.

[6] C. Deffayet, hep-th/0010186 (to appear in Phys. Lett. B), and references therein.

[7] C. Csaki, M. Graesser, L. Randall, J. Terning, Phys. Rev. D 62, 045015 (2000) hep-ph/9911406.

[8] P. Kanti, I. I. Kogan, K. A. Olive and M. Pospelov, Phys. Lett. B468 (1999) 31 hep-ph/9909481; P. Kanti, I. I. Kogan, K. A. Olive and M. Pospelov, Phys. Rev. D 61 (2000) 106004 hep-ph/9912266.

[9] C. Charmousis, R. Gregory, V. A. Rubakov, Phys. Rev. D 62, 067505 (2000) hep-th/9912160. 
[10] W.D. Goldberger, M.B. Wise, Phys. Rev. Lett. 83, 4922 (1999) hep-ph/9907447.

[11] O. DeWolfe, D. Z. Freedman, S. S. Gubser and A. Karch, Phys. Rev. D 62 (2000) 046008 hep-th/9909134.

[12] T. Tanaka, X. Montes, Nucl. Phys. B 582, 259 (2000) hepth/0001092.

[13] J. Garriga, O. Pujolas, T. Tanaka, "Radion effective potential in the Brane-World", hep-th/0004109.

[14] J. Garriga, T. Tanaka, Phys. Rev. Lett. 84, 2778 (2000).

[15] C. Csaki, M. Graesser, G.D. Kribs, "Radion Dynamics and Electroweak Physics", hep-th/0008151.

[16] J.M. Cline, H. Firouzjahi, Phys. Lett. B 495, 271 (2000) hepth/0008185.

[17] A. Mennim, R.A. Battye, "Cosmological expansion on a dilatonic brane-world", hep-th/0008192.

[18] N. Arkani-Hamed, M. Porrati, L. Randall, hep-th/0012148; R. Rattazzi, A. Zaffaroni, hep-th/0012248.

[19] P. Bowcock, C. Charmousis, R. Gregory, Class. Quant. Grav. 17, 4745 (2000).

[20] U. Gen, M. Sasaki, "Radion on the de Sitter brane", grqc/0011078

[21] A. Karch, L. Randall, "Locally Localized Gravity", hepth/0011156

[22] D. Langlois, "Evolution of cosmological perturbations in a braneuniverse", hep-th/0010063, to appear in Phys. Rev. Lett.

[23] W. Israel, Nuovo Cimento B44 (1966).

[24] Chanyong Park, Sang-Jin Sin, Phys. Lett. B 485, 239 (2000) hep-th/0005013.

[25] N. Kaloper, Phys. Rev. D 60, 123506 (1999) [hep-th/9905210.

[26] D. Langlois, R. Maartens, D. Wands, Phys. Lett. B 89, 259 (2000) hep-th/0006007.

[27] I. I. Kogan, S. Mouslopoulos and A. Papazoglou, Phys. Lett. B 501, 140 (2001) hep-th/0011141. 